\title{
One-dimensional infinitesimal-birational duality through differential operators
}

\author{
by \\ Tomasz Maszczyk (Warszawa)
}

\begin{abstract}
The structure of filtered algebras of Grothendieck's differential operators on a smooth fat point in a curve and graded Poisson algebras of their principal symbols is explicitly determined. A related infinitesimal-birational duality realized by a Springer type resolution of singularities and the Fourier transformation is presented. This algebrogeometrical duality is quantized in appropriate sense and its quantum origin is explained.
\end{abstract}

It seems that our ship is arriving at a coast, but the land is still hidden in the fog.

1. Introduction. The study of the algebra of differential operators of a commutative algebra was initiated by Grothendieck [11] in 1967. The most important question in this theory is how the properties of differential operators reflect the properties of a commutative algebra. Grothendieck proved that the algebra of differential operators on a smooth affine variety over a field of characteristic zero is generated by operators of order at most one [11], in particular is finitely generated. The converse, that generation by first order filtration implies smoothness, is now referred to as Nakai's conjecture. It implies the Zariski-Lipman conjecture [19], [1], [12]. Nakai's conjecture and its variants have been verified only for few classes of commutative algebras in [26], [13], [14], [27], [34], [20], [32], [31]. In general, the determination of the structure of differential operators of a given non-smooth commutative algebra is a hard problem and one expects some pathology caused by singularities. For example, the algebra of differential operators on the cubic

2000 Mathematics Subject Classification: Primary 16S32, 17B63; Secondary 16S30, $53 \mathrm{D} 20$.

Key words and phrases: algebraic differential operators, non-reduced schemes, moment map, Springer resolution, Fourier transform, Morita equivalence.

The author was partially supported by KBN grants 1P03A 03626 and 115/E343/SPB/6.PR UE/DIE 50/2005-2008. 
cone $x^{3}+y^{3}+z^{3}=0$ is not generated by operators of bounded order, in particular is not finitely generated [3]. Although for reduced curves the algebra of differential operators is Noetherian [21], [29], for the non-reduced commutative algebra of Krull dimension one, $k[x, y] /\left(x^{2}, x y\right)$, its algebra of differential operators is right but not left Noetherian [21]. For such problems, and other ones, concerning the structure of the algebra of differential operators, see also [4]-[7], [16]-[18], [21]-[25], [28]-[30], [33].

In this paper we determine the structure of the filtered algebra $\mathcal{D}\left(\mathcal{O}_{n}\right)$ of differential operators of the polynomial algebra $\mathcal{O}_{n}=\mathcal{O} / \mathrm{m}^{n+1}$ of the $n$th infinitesimal neighborhood of a smooth point in a curve over a field of characteristic zero, and its associated graded Poisson algebra $\mathcal{P}\left(\mathcal{O}_{n}\right)$. It turns out that instead of expected pathology we find beautiful and intriguing phenomena.

We can assume that our curve is the affine line, where $\mathcal{O}_{n}=k[x] /\left(x^{n+1}\right)$. Let $(e, h, f)$ be the standard basis of $\mathrm{sl}_{2}$, subject to the relations $[e, f]=h$, $[h, e]=2 e,[h, f]=-2 f$. We show that there exists an extension of filtered algebras

$$
\left(e^{n+1}\right) \longmapsto \mathcal{U}\left(\mathrm{sl}_{2}\right) /(C-n(n+2)) \rightarrow \mathcal{D}\left(\mathcal{O}_{n}\right),
$$

where $C=h^{2}+2(e f+f e)$ is the Casimir element and we filter the enveloping algebra giving $(e, h, f)$ orders $(0,1,2)$. The epimorphism maps $(e, h, f)$ to

$$
\left(x, 2 x \frac{d}{d x}-n,-x \frac{d^{2}}{d x^{2}}+n \frac{d}{d x}\right) .
$$

In particular, $\mathcal{D}\left(\mathcal{O}_{n}\right)$ for every $n$ is generated by operators of order less than or equal 2 , or more precisely, by $x$ and a single operator of order 2 . This means that the algebras $\mathcal{O}_{n}$ of the $n$th infinitesimal neighborhoods of a one-dimensional smooth point are close to smooth algebras.

After passing to the associated graded Poisson algebras we get the extension

$$
\mathfrak{m}^{n+1} \longmapsto \mathcal{P} \rightarrow \mathcal{P}\left(\mathcal{O}_{n}\right),
$$

where $\mathcal{P}$ is the algebra of polynomial functions on the nilpotent cone in $\mathrm{sl}_{2}^{*}$ with its canonical graded Kirillov-Kostant Poisson structure and $\mathfrak{m}$ is the maximal ideal of the vertex. Both extensions are $\mathrm{sl}_{2}$-invariant in an appropriate sense. In the second extension the epimorphism describes the closed embedding via the moment map associated with a hamiltonian action of $\operatorname{sl}_{2}$ on $\operatorname{Spec}\left(\mathcal{P}\left(\mathcal{O}_{n}\right)\right)$.

Although for $n>0$ surjective homomorphisms of the commutative algebras $\mathcal{O}_{n+1} \rightarrow \mathcal{O}_{n}$ do not induce homomorphisms of the algebras of differential operators $\mathcal{D}\left(\mathcal{O}_{n+1}\right) \rightarrow \mathcal{D}\left(\mathcal{O}_{n}\right)$ (there is no homomorphism of algebras of matrices $\mathcal{M}_{n+1} \rightarrow \mathcal{M}_{n}$ ), we have surjective homomorphisms of their graded Poisson algebras $\mathcal{P}\left(\mathcal{O}_{n+1}\right) \rightarrow \mathcal{P}\left(\mathcal{O}_{n}\right)$, which is a bit of a surprise. 
Next, we introduce the notion of quantization of graded Poisson algebras and show that the first extension can be regarded as a quantization of the second extension of their associated commutative graded Poisson algebras. Although maximal primitive quotients of the enveloping algebra $\mathcal{U}\left(\mathrm{sl}_{2}\right) /(C-n(n+2))$ depend on $n$, they are Morita equivalent to one another by the Beilinson-Bernstein theorem [2]. It is easy to see that forgetting about filtration one gets $\mathcal{D}\left(\mathcal{O}_{n}\right) \cong \operatorname{End}_{k}\left(\mathcal{O}_{n}\right)$ so for all $n$ the algebras $\mathcal{D}\left(\mathcal{O}_{n}\right)$ are Morita equivalent, i.e. the respective categories of $\mathcal{D}$-modules are equivalent to one another. This means that although quantizations of all infinitesimal neighborhoods of the vertex of the nilpotent cone are not related by homomorphisms, they are related at least by combinations of homomorphisms at the level of commutative (graded Poisson) algebras and Morita equivalences at the level of noncommutative (almost commutative) algebras.

Next, after passing to inverse limits, we compare the completion of the algebra of principal symbols on the affine line with the inverse limit of the above system of algebras of principal symbols on infinitesimal neighborhoods of a point in the affine line. We show that there exists a unique formal grading preserving homomorphism of formally graded Poisson algebras over the completed local ring of a point in the line, between these two limits. Geometrically, this is a completion of a resolution of singularities in the category of conical Poisson varieties

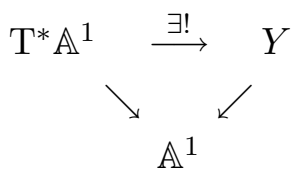

Finally, we show that the above canonical resolution of singularity together with the Fourier transformation establishes a duality between the above $\mathrm{SL}_{2}$-symmetries of principal symbols of the system of infinitesimal neighborhoods of finite order of a point in the line $\mathbb{A}^{1}$ (infinitesimal picture) and automorphisms of dual principal symbols induced by birational automorphisms of the dual line $\widehat{\mathbb{A}}^{1}$ (birational picture).

Magic Diamond Theorem. The following diagram of canonical morphisms in the category of conical Poisson varieties is commutative:

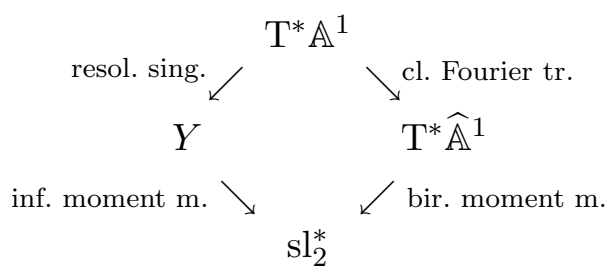

This diagram can be quantized in the above sense, which relates noncommutative algebra and commutative algebraic geometry as follows: 
Quantized Magic Diamond Theorem. There exists the following commutative diagram in the category of almost commutative algebras:

$$
\begin{aligned}
& \text { q. resol. sing. } \nearrow^{\mathcal{D}\left(\mathbb{A}^{1}\right)} \nwarrow^{\text {q. Fourier tr. }} \\
& \mathcal{U}\left(\mathrm{sl}_{2}\right) /(C) \quad \mathcal{D}\left(\widehat{\mathbb{A}}^{1}\right) \\
& \text { q. inf. moment m. } \backslash \mathcal{U}\left(\mathrm{sl}_{2}\right) \nearrow \text { q. bir. moment m. }
\end{aligned}
$$

which is a quantization of the Magic Diamond.

A more conceptual explanation of unexpected relations between the following facets of our example:

- $\mathcal{D}$-modules on non-reduced schemes,

- representation theory and moment maps,

- resolution of singularities,

- Fourier transformation,

- Morita equivalence,

would be desirable.

Acknowledgements. I would like to express my gratitude to Mariusz Wodzicki for numerous helpful discussions and encouragement.

2. Basic definitions. Let $k$ be a field of characteristic zero.

Definition 1. A filtered associative algebra $\mathcal{D}=\bigcup_{p \geq 0} \mathcal{D}^{p}, \mathcal{D}^{p} \subset \mathcal{D}^{p+1}$, is called almost commutative if $\left[\mathcal{D}^{p}, \mathcal{D}^{q}\right] \subset \mathcal{D}^{p+q-1}$.

Definition 2. Given a commutative algebra $\mathcal{O}$ one defines the filtered algebra $\mathcal{D}(\mathcal{O})=\bigcup_{p \geq 0} \mathcal{D}^{p}(\mathcal{O})$ of differential operators of $\mathcal{O}$, where

$$
\mathcal{D}^{p}(\mathcal{O}):=\left\{\delta \in \operatorname{End}_{k}(\mathcal{O}) \mid \forall_{f_{0}, \ldots, f_{p} \in \mathcal{O}}\left[f_{0}, \ldots,\left[f_{p}, \delta\right] \ldots\right]=0\right\} .
$$

This filtration is called the order filtration.

One can show that the filtered associative algebra $\mathcal{D}(\mathcal{O})$ is almost commutative.

Definition 3. A graded commutative algebra $\mathcal{P}=\bigoplus_{p \geq 0} \mathcal{P}^{p}$ is called a graded Poisson algebra if there is a Lie algebra structure on $\mathcal{P}$,

$$
\{-,-\}: \mathcal{P} \otimes \mathcal{P} \rightarrow \mathcal{P},
$$

such that

$$
\left\{\mathcal{P}^{p}, \mathcal{P}^{q}\right\} \subset \mathcal{P}^{p+q-1}
$$

and for all $f, g, h \in \mathcal{P}$,

$$
\{f, g h\}=\{f, g\} h+g\{f, h\} .
$$


ExAmPLE. The associated graded commutative algebra $\operatorname{Gr}(\mathcal{D}):=$ $\bigoplus_{p \geq 0} \mathcal{D}^{p} / \mathcal{D}^{p-1}$ of an almost commutative filtered algebra $\mathcal{D}$ is a graded Poisson algebra, where for all $\delta \in \mathcal{D}^{p}$ and $\varepsilon \in \mathcal{D}^{q}$,

$$
\left\{\delta+\mathcal{D}^{p-1}, \varepsilon+\mathcal{D}^{q-1}\right\}:=[\delta, \varepsilon]+\mathcal{D}^{p+q-2} .
$$

Definition 4. Homogeneous elements of the graded Poisson algebra $\mathcal{P}(\mathcal{O}):=\operatorname{Gr}(\mathcal{D}(\mathcal{O}))$ are called principal symbols of differential operators of $\mathcal{O}$.

For $X=\operatorname{Spec}(\mathcal{O})$ we will write $\mathcal{D}(X):=\mathcal{D}(\mathcal{O}), \mathcal{P}(X):=\mathcal{P}(\mathcal{O})$.

EXAMPLE. Let $\mathfrak{g}=\bigcup_{p \geq 0} \mathfrak{g}^{p}, \mathfrak{g}^{p} \subset \mathfrak{g}^{p+1}$, be a filtered Lie algebra, i.e.

$$
\left[\mathfrak{g}^{p}, \mathfrak{g}^{q}\right] \subset \mathfrak{g}^{p+q-1} .
$$

Let us give its enveloping algebra $\mathcal{U}(\mathfrak{g})$ the induced filtration. Then $\mathcal{U}(\mathfrak{g})$ becomes an almost commutative algebra whose associated graded Poisson algebra $\operatorname{Gr}(\mathcal{U}(\mathfrak{g}))$ is the symmetric algebra $\mathrm{S}(\operatorname{Gr}(\mathfrak{g}))$ of the associated graded vector space $\operatorname{Gr}(\mathfrak{g})=\bigoplus_{p \geq 0} \mathfrak{g}^{p} / \mathfrak{g}^{p-1}$, where for generators $X \in \mathfrak{g}^{p}, Y \in \mathfrak{g}^{q}$,

$$
\left\{X+\mathfrak{g}^{p-1}, Y+\mathfrak{g}^{q-1}\right\}:=[X, Y]+\mathfrak{g}^{p+q-2} .
$$

Any ad-invariant homogeneous ideal in $\mathrm{S}(\mathrm{Gr}(\mathfrak{g}))$ defines a graded Poisson structure on the factor-algebra. One calls it the graded Kirillov-Kostant Poisson structure.

Definition 5. For a given conical Poisson $\operatorname{scheme} \operatorname{Spec}(\mathcal{P})$ its quantization is a pair consisting of an almost commutative algebra $\mathcal{D}$ and an isomorphism of graded Poisson algebras $\operatorname{Gr}(\mathcal{D}) \rightarrow \mathcal{P}$.

For a given morphism of conical Poisson schemes $\operatorname{Spec}\left(\mathcal{P}_{1}\right) \rightarrow \operatorname{Spec}\left(\mathcal{P}_{2}\right)$ its quantization is a pair consisting of a filtration preserving homomorphism of almost commutative algebras $\mathcal{D}_{1} \leftarrow \mathcal{D}_{2}$ and a commutative diagram in the category of graded Poisson algebras

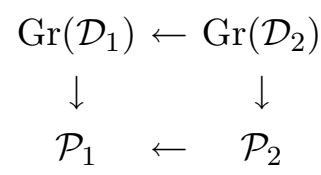

with canonical horizontal arrows and vertical arrows being isomorphisms. The definition of a quantization of an arbitrary diagram in the category of conical Poisson schemes is obvious.

Note that any almost commutative algebra (resp. any filtration preserving homomorphism of almost commutative algebras) is a quantization of the spectrum of its associated graded Poisson algebra (resp. of the induced morphism between associated conical Poisson schemes). Another example of quantization arises in a canonical way in the following context. 
Definition 6. A morphism of conical Poisson schemes

$$
\operatorname{Spec}(\mathcal{P}) \rightarrow \operatorname{Spec}(\operatorname{S}(\operatorname{Gr}(\mathfrak{g})))
$$

determined by a homomorphism of graded Poisson algebras

$$
\mathcal{P} \leftarrow \mathrm{S}(\operatorname{Gr}(\mathfrak{g}))
$$

is called the moment map.

EXAMPLE. Let $\mathcal{O}$ be a commutative algebra acted on by a filtered Lie algebra $\mathfrak{g}$ through differential operators. Assume that the filtration on $\mathfrak{g}$ is compatible with the order filtration on $\mathcal{D}(\mathcal{O})$. Then we obtain a homomorphism of almost commutative algebras

$$
\mathcal{D}(\mathcal{O}) \leftarrow \mathcal{U}(\mathfrak{g}),
$$

which is a quantization of the induced moment map

$$
\operatorname{Spec}(\mathcal{P}(\mathcal{O})) \rightarrow \operatorname{Spec}(\operatorname{S}(\operatorname{Gr}(\mathfrak{g}))) .
$$

Note that the set of $k$-points of $\operatorname{Spec}(\operatorname{S}(\operatorname{Gr}(\mathfrak{g})))$ can be canonically identified with the dual space $\operatorname{Gr}(\mathfrak{g})^{*}$. Since all our structures are defined over $k$ we will abuse the language and will not distinguish $\operatorname{Spec}(\operatorname{S}(\operatorname{Gr}(\mathfrak{g})))$ from $\operatorname{Gr}(\mathfrak{g})^{*}$. In the case of a filtered Lie algebra coming from a graded Lie algebra $\mathfrak{g}=\bigoplus_{i \geq 0} \mathfrak{g}_{i},\left[\mathfrak{g}_{i}, \mathfrak{g}_{j}\right] \subset \mathfrak{g}_{i+j-1}$, i.e. when the filtration has the form $\mathfrak{g}^{p}=\bigoplus_{0 \leq i \leq p} \mathfrak{g}_{i}$, we will not distinguish also the graded vector space $\operatorname{Gr}(\mathfrak{g})$ from the graded Lie algebra $\mathfrak{g}$.

If one gives a commutative algebra $\mathcal{O}$ the trivial grading and the trivial Poisson structure then one has the canonical injective homomorphism of graded Poisson algebras $\mathcal{O} \longmapsto \mathcal{P}(\mathcal{O})$ onto the subalgebra of symbols of degree zero, and the canonical surjective homomorphism of graded $\mathcal{O}$-algebras $\mathcal{P}(\mathcal{O}) \rightarrow \mathcal{O}$ annihilating all symbols of positive degree. If $\mathcal{O}=\mathcal{O}(V)$ is the algebra of polynomial functions on a smooth affine variety $V$ then $\operatorname{Spec}(\mathcal{P}(\mathcal{O}))=\mathrm{T}^{*} V$, the cotangent bundle of $V$. Then the above homomorphisms describe the canonical projection $\mathrm{T}^{*} V \rightarrow V$ and the embedding onto the zero section $V \rightarrow \mathrm{T}^{*} V$, respectively.

Let $E$ be a finite-dimensional graded vector space and $\widehat{E}=E^{*}[1]$ be its dual with the dual grading enlarged by one. Then

$$
\mathrm{T}^{*} \operatorname{Spec}(\mathrm{S}(E))=\operatorname{Spec}(\mathrm{S}(E) \otimes \mathrm{S}(\widehat{E}))
$$

is a conical Poisson variety with the following Poisson structure:

$$
\left\{e_{1} \otimes 1, e_{2} \otimes 1\right\}=0, \quad\left\{1 \otimes \widehat{e}_{1}, 1 \otimes \widehat{e}_{2}\right\}=0, \quad\{1 \otimes \widehat{e}, e \otimes 1\}=\widehat{e}(e) .
$$

Using the canonical linear isomorphism of graded vector spaces $E \rightarrow \widehat{\widehat{E}}$, we obtain the canonical identification

$$
\mathrm{T}^{*} \operatorname{Spec}(\mathrm{S}(\widehat{E})) \stackrel{\cong}{\rightarrow} \operatorname{Spec}(\mathrm{S}(\widehat{E}) \otimes \mathrm{S}(E)) \text {. }
$$


Definition 7. We define an isomorphism of conical Poisson varieties

$$
\mathrm{T}^{*} \operatorname{Spec}(\mathrm{S}(E)) \rightarrow \mathrm{T}^{*} \operatorname{Spec}(\mathrm{S}(\widehat{E}))
$$

by means of polynomial functions as follows:

$$
\begin{gathered}
\mathrm{S}(E) \otimes \mathrm{S}(\widehat{E}) \leftarrow \mathrm{S}(\widehat{E}) \otimes \mathrm{S}(E), \\
\widehat{e} \otimes 1 \mapsto 1 \otimes \widehat{e}, \quad 1 \otimes e \mapsto-e \otimes 1 .
\end{gathered}
$$

We call this isomorphism the classical Fourier transformation.

According to Definition 5 the quantum Fourier transformation

$$
\mathcal{D}\left(\widehat{\mathbb{A}}^{n}\right) \rightarrow \mathcal{D}\left(\mathbb{A}^{n}\right), \quad \widehat{x}_{i} \mapsto \frac{\partial}{\partial x_{i}}, \frac{\partial}{\partial \widehat{x}_{i}} \mapsto-x_{i},
$$

is a quantization of the classical Fourier transformation, provided $\mathcal{D}\left(\widehat{\mathbb{A}}^{n}\right)$ is filtered not by the order of differential operators but by the degree of their polynomial coefficients. Whenever $\mathcal{D}\left(\widehat{\mathbb{A}}^{n}\right)$ appears in this article, it is equipped with this last filtration, making it an almost commutative algebra. Then the classical Fourier transformation on the level of cotangent bundles $\mathrm{T}^{*}\left(\mathbb{A}^{n}\right) \rightarrow \mathrm{T}^{*}\left(\widehat{\mathbb{A}}^{n}\right)$ can be regarded as a result of descending of the quantum Fourier transformation to the level of associated graded Poisson algebras, which justifies our terminology.

For the affine line $\mathbb{A}^{1}=\mathbb{P}^{1} \backslash\{\infty\}$ we can define the dual line as $\widehat{\mathbb{A}}^{1}=$ $\widehat{\mathbb{P}}^{1} \backslash\{\infty\}=\mathbb{P}^{1} \backslash\{0\}$, by self-duality of the projective line, and we regard $\mathrm{T}^{*} \widehat{\mathbb{A}}^{1}$ as an open subscheme in $\mathrm{T}^{*} \widehat{\mathbb{P}}^{1}$. The affine algebraic group $\mathrm{SL}_{2}$ acts on $\widehat{\mathbb{P}}^{1}$ and this action lifts canonically to the conical structure preserving Poisson action on the conical Poisson variety $\mathrm{T}^{*} \widehat{\mathbb{P}}^{1}$. We can regard the restriction of this action to $\mathrm{T}^{*} \widehat{\mathbb{A}}^{1}$ as the induced rational action of the pseudogroup of birational automorphisms of $\widehat{\mathbb{A}}^{1}$. The Lie algebra $\mathrm{sl}_{2}$ of vector fields on $\widehat{\mathbb{P}}^{1}$ lifts canonically to $\mathrm{T}^{*} \widehat{\mathbb{P}}^{1}$ and defines a moment map $\mathrm{T}^{*} \widehat{\mathbb{P}}^{1} \rightarrow \mathrm{sl}_{2}^{*}$. The restriction of this moment map to $\mathrm{T}^{*} \widehat{\mathbb{A}}^{1}$ can be understood as the moment map $\mathrm{T}^{*} \widehat{\mathbb{A}}^{1} \rightarrow \mathrm{sl}_{2}^{*}$ defined by the induced rational action of the pseudogroup of birational automorphisms of $\widehat{\mathbb{A}}^{1}$.

3. Differential operators of truncated polynomials. We will study the polynomial algebra $\mathcal{O}_{n}:=k[x] /\left(x^{n+1}\right)$ of the $n$th infinitesimal neighborhood $\mathbb{A}_{n}^{1}$ of a point $(x=0)$ on the affine line $\mathbb{A}^{1}$, the algebra $\mathcal{D}\left(\mathcal{O}_{n}\right)$ of differential operators on this neighborhood, and the associated graded Poisson algebra $\mathcal{P}\left(\mathcal{O}_{n}\right)$ spanned by their principal symbols. We identify functions from $\mathcal{O}_{n}$ with operators of order zero from $\mathcal{D}^{0}\left(\mathcal{O}_{n}\right)$ and with their principal symbols from $\mathcal{P}^{0}\left(\mathcal{O}_{n}\right)$, for instance we identify $x^{l} \in \mathcal{O}_{n}$ with the operator $\delta_{0}^{l} \in \mathcal{D}^{0}\left(\mathcal{O}_{n}\right)$ of multiplication by $x^{l}$ and with its principal symbol $y_{0}^{l} \in \mathcal{P}^{0}\left(\mathcal{O}_{n}\right)$. For any $\delta \in \mathcal{D}\left(\mathcal{O}_{n}\right)$ and $f \in \mathcal{O}_{n}$ we use the notation

$$
\operatorname{ad}_{f(x)}(\delta):=\left[f\left(\delta_{0}\right), \delta\right] \text {. }
$$


Lemma 1.

$$
\operatorname{ad}_{x}^{p+1}(\delta)=0 \Leftrightarrow \forall_{f_{0}, \ldots, f_{p} \in \mathcal{O}_{n}} \operatorname{ad}_{f_{0}} \cdots \operatorname{ad}_{f_{p}}(\delta)=0 .
$$

Proof. One can assume $f_{0}=x^{l_{0}}, \ldots, f_{p}=x^{l_{p}}$ for $l_{0}, \ldots, l_{p} \geq 0$. Since $\operatorname{ad}_{x^{l}}(\delta)=\sum_{m=1}^{l} \delta_{0}^{m-1} \operatorname{ad}_{x}(\delta) \delta_{0}^{l-m}$ and $\operatorname{ad}_{x}$ commutes with left and right multiplication by $\delta_{0}$ we get

$$
\begin{aligned}
\operatorname{ad}_{x^{l_{0}}} \cdots \operatorname{ad}_{x^{l_{p}}}(\delta) & \\
& =\sum_{v_{0}=1}^{l_{0}} \delta_{0}^{v_{0}-1} \operatorname{ad}_{x}\left(\sum_{v_{1}=1}^{l_{1}} \delta_{0}^{v_{1}-1} \operatorname{ad}_{x}\left(\cdots \delta_{0}^{v_{p}-1} \operatorname{ad}_{x}(\delta) \delta_{0}^{l_{p}-v_{p}}\right) \cdots\right) \delta_{0}^{l_{0}-v_{0}} \\
& =\sum_{v_{0}=1}^{l_{0}} \cdots \sum_{v_{p}=1}^{l_{p}} \delta_{0}^{\left(v_{0}-1\right)+\cdots+\left(v_{p}-1\right)} \operatorname{ad}_{x}^{p+1}(\delta) \delta_{0}^{\left(l_{0}-v_{0}\right)+\cdots+\left(l_{p}-v_{p}\right)}
\end{aligned}
$$

which implies the lemma.

Lemma 2.

$$
x^{n+1}=0 \Rightarrow \operatorname{ad}_{x}^{2 n+1}=0 .
$$

Proof. Substituting $l=2 n+1$ into the identity

$$
\operatorname{ad}_{x}^{l}(\delta)=\sum_{m=0}^{p}(-1)^{m+1}\left(\begin{array}{c}
l \\
m
\end{array}\right) \delta_{0}^{l-m} \delta \delta_{0}^{m}
$$

one can see that at least one of $m, l-m$ is greater than $n$.

Corollary 1.

$$
\mathcal{D}\left(\mathcal{O}_{n}\right)=\operatorname{End}_{k}\left(\mathcal{O}_{n}\right)
$$

COROLlary 2. The order filtration on differential operators has the form

$$
\mathcal{D}^{p}\left(\mathcal{O}_{n}\right)=\left\{\delta \in \operatorname{End}_{k}\left(\mathcal{O}_{n}\right) \mid \operatorname{ad}_{x}^{p+1}(\delta)=0\right\} .
$$

Lemma 3. We have the canonical linear embedding $\mathcal{P}^{p}\left(\mathcal{O}_{n}\right) \longmapsto \mathcal{O}_{n}$ induced by the map $\operatorname{ad}_{x}^{p}: \mathcal{D}^{p}\left(\mathcal{O}_{n}\right) \rightarrow \mathcal{O}_{n}$. It is multiplicative in the following sense: for all $\delta \in \mathcal{D}^{p}\left(\mathcal{O}_{n}\right)$ and $\varepsilon \in \mathcal{D}^{q}\left(\mathcal{O}_{n}\right)$,

$$
\frac{1}{(p+q) !} \operatorname{ad}_{x}^{p+q}(\delta \varepsilon)=\frac{1}{p !} \operatorname{ad}_{x}^{p}(\delta) \frac{1}{q !} \operatorname{ad}_{x}^{q}(\varepsilon) .
$$

Proof. Since $\mathcal{D}^{p}\left(\mathcal{O}_{n}\right)=\operatorname{ker~ad}_{x}^{p+1}$, it follows that $\operatorname{ad}_{x}^{p}$ maps $\mathcal{D}^{p}\left(\mathcal{O}_{n}\right)$ into $\mathcal{O}_{n}=\operatorname{kerad}_{x}$ with kernel $\mathcal{D}^{p-1}\left(\mathcal{O}_{n}\right) \subset \mathcal{D}^{p}\left(\mathcal{O}_{n}\right)$, hence embeds $\mathcal{D}^{p}\left(\mathcal{O}_{n}\right)=$ $\mathcal{D}^{p}\left(\mathcal{O}_{n}\right) / \mathcal{D}^{p-1}\left(\mathcal{O}_{n}\right)$ into $\mathcal{O}_{n}$. Using the fact that $\operatorname{ad}_{x}^{l}(\delta)=0$ and $\operatorname{ad}_{x}^{m}\left(\delta^{\prime}\right)=0$ for $l>p$ and $m>q$ in the identity

$$
\frac{1}{(p+q) !} \operatorname{ad}_{x}^{p+q}(\delta \varepsilon)=\sum_{l+m=p+q} \frac{1}{l !} \operatorname{ad}_{x}^{l}(\delta) \frac{1}{m !} \operatorname{ad}_{x}^{m}(\varepsilon)
$$

we get on the right hand side only one summand with $l=p$ and $m=q$. 
Note that $\operatorname{ad}_{x}^{p}: \mathcal{D}^{p}\left(\mathcal{O}_{n}\right) \rightarrow \mathcal{O}_{n}$ is $\mathcal{O}_{n}$-linear, hence its image is an ideal, necessarily generated by $x^{v_{p}}$ for some $v_{p} \leq n$.

Definition 8. We define operators $\delta_{p} \in \mathcal{D}^{p}\left(\mathcal{O}_{n}\right)$ for $p=1, \ldots, 2 n$ such that

$$
\frac{1}{p !} \operatorname{ad}_{x}^{p}\left(\delta_{p}\right)=\delta_{0}^{v_{p}}
$$

Note that the operators $\delta_{p}$ are determined up to $\mathcal{D}^{p-1}\left(\mathcal{O}_{n}\right)=\operatorname{ker}\left(\operatorname{ad}_{x}^{p}\right)$. However, by Lemma 3, their principal symbols $y_{p} \in \mathcal{P}^{p}\left(\mathcal{O}_{n}\right)$ are defined uniquely.

Lemma 4. The system $\left(y_{0}^{l} y_{p}\right)_{l=0}^{n-v_{p}}$ is a basis of $\mathcal{P}^{p}\left(\mathcal{O}_{n}\right)$ for $i=1, \ldots, 2 n$.

Proof. Since on the right hand side of the equality

$$
\frac{1}{p !} \operatorname{ad}_{x}^{p}\left(\delta_{0}^{l} \delta_{p}\right)=\delta_{0}^{l+v_{p}}
$$

we get linearly independent powers of $\delta_{0}$, the system of the corresponding principal symbols is linearly independent in $\mathcal{P}^{p}\left(\mathcal{O}_{n}\right)$. On the other hand, for every $\delta \in \mathcal{D}^{p}\left(\mathcal{O}_{n}\right)$ we have the decomposition

$$
\begin{aligned}
\operatorname{ad}_{x}^{p}(\delta) & =\sum_{l=0}^{n-v_{p}} c_{l} \delta_{0}^{l+v_{p}}=\sum_{l=0}^{n-v_{p}} c_{l} \delta_{0}^{l} \delta_{0}^{v_{p}}=\sum_{l=0}^{n-v_{p}} c_{l} \delta_{0}^{l} \frac{1}{p !} \operatorname{ad}_{x}^{p}\left(\delta_{p}\right) \\
& =\operatorname{ad}_{x}^{p}\left(\sum_{l=0}^{n-v_{p}} \frac{c_{l}}{p !} \delta_{0}^{l} \delta_{p}\right)
\end{aligned}
$$

which means that

$$
\delta \equiv \sum_{l=0}^{n-v_{p}} \frac{c_{l}}{p !} \delta_{0}^{l} \delta_{p} \bmod \mathcal{D}^{p-1}\left(\mathcal{O}_{n}\right) .
$$

Therefore the above system of principal symbols generates $\mathcal{P}^{p}\left(\mathcal{O}_{n}\right)$.

Corollary 3.

$$
\operatorname{dim} \mathcal{P}^{p}\left(\mathcal{O}_{n}\right)=n-v_{p}+1
$$

Corollary 4.

$$
v_{1}+\cdots+v_{2 n}=n(n+1)
$$

Proof. We have

$$
\begin{aligned}
& (n+1)^{2}=\operatorname{dim} \operatorname{End}_{k}\left(\mathcal{O}_{n}\right)=\operatorname{dim} \mathcal{D}\left(\mathcal{O}_{n}\right) \\
& \quad=\operatorname{dim} \mathcal{O}_{n}+\sum_{p=1}^{2 n} \operatorname{dim} \mathcal{P}^{p}\left(\mathcal{O}_{n}\right)=(n+1)+2 n(n+1)-\left(v_{1}+\cdots+v_{2 n}\right) .
\end{aligned}
$$

Our next task is to determine $v_{p}$ 's for $i=1, \ldots, 2 n$.

LEMma 5. $v_{p}>0$. 
Proof. In the ordered basis $\left(1, x, \ldots, x^{n}\right)$ of $\mathcal{O}_{n}$ multiplication by $x$ has the form of a subdiagonal Jordan block. Therefore we have

$$
\operatorname{tr}\left(\delta_{0}^{v_{p}}\right)=\frac{1}{p !} \operatorname{tr}\left(\operatorname{ad}_{x}^{p}\left(\delta_{p}\right)\right)=0
$$

But this is possible only if $v_{p}>0$.

Lemma 6. If $n>0$ then $v_{1}=v_{2}=1$.

Proof. We already know that $v_{1}, v_{2} \geq 1$. We show that $v_{1}, v_{2} \leq 1$. Using the Jordan form we can easily find a solution $\delta_{2} \in \operatorname{End}_{k}\left(\mathcal{O}_{n}\right)$ to the equation

$$
\frac{1}{2} \operatorname{ad}_{x}^{2}\left(\delta_{2}\right)=\delta_{0}
$$

Therefore $v_{2}=1$. If we take $\delta_{1}:=\frac{1}{2} \operatorname{ad}_{x}\left(\delta_{2}\right)$ then

$$
\operatorname{ad}_{x}\left(\delta_{1}\right)=\frac{1}{2} \operatorname{ad}_{x}^{2}\left(\delta_{2}\right)=\delta_{0}
$$

Therefore $v_{1}=1$.

One can easily check that one can choose the operator $\delta_{2}$ of the form $x d^{2} / d x^{2}-n d / d x$. Then the operators

$$
\delta_{0}:=x, \quad \delta_{1}:=-x \frac{d}{d x}+\frac{n}{2}, \quad \delta_{2}:=x \frac{d^{2}}{d x^{2}}-n \frac{d}{d x}
$$

satisfy the relations

$$
\left[\delta_{0}, \delta_{1}\right]=\delta_{0}, \quad\left[\delta_{0}, \delta_{2}\right]=2 \delta_{1}, \quad\left[\delta_{1}, \delta_{2}\right]=\delta_{2},
$$

and

$$
\delta_{1}^{2}-\frac{1}{2}\left(\delta_{0} \delta_{2}+\delta_{2} \delta_{0}\right)=\frac{n}{2}\left(\frac{n}{2}+1\right),
$$

which means that we have a homomorphism of filtered algebras from a maximal primitive quotient of the enveloping algebra of $\mathrm{sl}_{2}$ (see [10]) into $\mathcal{D}\left(\mathcal{O}_{n}\right)$

$$
\begin{aligned}
& \mathcal{U}\left(\mathrm{sl}_{2}\right) /(C-n(n+2)) \rightarrow \mathcal{D}\left(\mathcal{O}_{n}\right), \\
& e \mapsto \delta_{0}, \quad h \mapsto-2 \delta_{1}, \quad f \mapsto-\delta_{2},
\end{aligned}
$$

where $\mathrm{sl}_{2}$ is spanned by $(e, h, f)$ subject to the relations

$$
[e, f]=h, \quad[h, e]=2 e, \quad[h, f]=-2 f,
$$

and $C$ denotes the Casimir element $h^{2}+2(e f+f e)$.

Definition 9. On the maximal primitive quotient we define a filtration coming from the filtration on the Lie algebra $\mathrm{sl}_{2}:(e) \subset(e, h) \subset(e, h, f)=$ $\mathrm{sl}_{2}$. We call the above choice (5) of $\delta_{0}, \delta_{1}, \delta_{2}$ and the resulting homomorphism (8) of filtered algebras distinguished. 
Note that this filtration differs from the standard filtration of the enveloping algebra of a Lie algebra, under which the Lie algebra lies entirely in the first piece of the filtration.

Lemma 7. If $n>0$ then $v_{2 l-1}=v_{2 l}=l$ for $l=1, \ldots, n$.

Proof. Note first that if $x^{l} \in \operatorname{ad}_{x}^{p}\left(\mathcal{D}\left(\mathcal{O}_{n}\right)^{p}\right) \subset \mathcal{O}_{n}$ then $v_{p} \leq l$. Using the multiplicative law from Lemma 3 and the equalities $v_{1}=v_{2}=1$ we get

$$
\begin{aligned}
\frac{1}{(2 l) !} \operatorname{ad}_{x}^{2 l}\left(\delta_{2}^{l}\right) & =\left(\frac{1}{2} \operatorname{ad}_{x}^{2}\left(\delta_{2}\right)\right)^{l}=\delta_{0}^{l}, \\
\frac{1}{(2 l-1) !} \operatorname{ad}_{x}^{2 l-1}\left(\delta_{1} \delta_{2}^{l-1}\right) & =\operatorname{ad}_{x}\left(\delta_{1}\right)\left(\frac{1}{2} \operatorname{ad}_{x}^{2}\left(\delta_{2}\right)\right)^{l-1}=\delta_{0}^{l},
\end{aligned}
$$

which means that $v_{2 l-1}, v_{2 l} \leq l$. But on the other hand, $v_{2 l-1}, v_{2 l} \geq 0$ and

$$
n(n+1)=v_{1}+\cdots+v_{2 n}=\sum_{l=1}^{n}\left(v_{2 l-1}+v_{2 l}\right) \leq \sum_{l=1}^{n}(l+l)=n(n+1),
$$

which implies the desired equalities.

Definition 10. We will identify the graded algebras $\mathrm{S}\left(\mathrm{sl}_{2}\right)$ and $k\left[z_{0}, z_{1}, z_{2}\right]$ using

$$
z_{0}=e, \quad z_{1}=-\frac{1}{2} h, \quad z_{2}=-f,
$$

where $\operatorname{deg} z_{p}=p$. Then the canonical Kirillov-Kostant Poisson structure takes the following form:

$$
\left\{z_{0}, z_{1}\right\}=z_{0}, \quad\left\{z_{0}, z_{2}\right\}=2 z_{1}, \quad\left\{z_{1}, z_{2}\right\}=z_{2} .
$$

Let $\mathcal{P}:=k\left[z_{0}, z_{1}, z_{2}\right] /\left(z_{1}^{2}-z_{0} z_{2}\right)$ be a graded polynomial algebra of the Zariski closure of the unique non-closed $\mathrm{Ad}_{\mathrm{SL}_{2}}^{*}$-orbit in sl ${ }_{2}^{*}$ (which here can be identified via the Killing form with the nilpotent cone in $\mathrm{sl}_{2}$ ).

The maximal ideal of the vertex $\mathfrak{m}=\left(z_{0}, z_{1}, z_{2}\right)$ is a homogeneous Poisson ideal. We set $\mathcal{P}_{n}:=\mathcal{P} / \mathfrak{m}^{n+1}$.

TheOREM 1. There exists an isomorphism of graded polynomial Poisson algebras

$$
\mathcal{P}_{n} \rightarrow \mathcal{P}\left(\mathcal{O}_{n}\right), \quad\left(z_{0}, z_{1}, z_{2}\right) \mapsto\left(y_{0}, y_{1}, y_{2}\right) .
$$

Proof. By Lemma 4 we know that the algebra $\mathcal{P}\left(\mathcal{O}_{n}\right)$ is generated by $y_{p}$ 's for $p=0, \ldots, 2 n$. By (9), (10) from the proof of Lemma 7, we have

$$
\begin{aligned}
\frac{1}{(2 l) !} \operatorname{ad}_{x}^{2 l}\left(\delta_{2}^{l}\right) & =\delta_{0}^{l}=\frac{1}{(2 l) !} \operatorname{ad}_{x}^{2 l}\left(\delta_{2 l}\right), \\
\frac{1}{(2 l-1) !} \operatorname{ad}_{x}^{2 l-1}\left(\delta_{1} \delta_{2}^{l-1}\right) & =\delta_{0}^{l}=\frac{1}{(2 l-1) !} \operatorname{ad}_{x}^{2 l-1}\left(\delta_{2 l-1}\right),
\end{aligned}
$$


which means that in $\mathcal{P}\left(\mathcal{O}_{n}\right)$,

$$
y_{2 l}=y_{2}^{l}, \quad y_{2 l-1}=y_{1} y_{2}^{l-1} .
$$

Therefore $\mathcal{P}\left(\mathcal{O}_{n}\right)$ is generated by $\left(y_{0}, y_{1}, y_{2}\right)$. By the multiplicative law (1) from Lemma 3, we have

$$
\begin{gathered}
\frac{1}{2} \operatorname{ad}_{x}^{2}\left(\delta_{1}^{2}\right)=\left(\operatorname{ad}_{x}\left(\delta_{1}\right)\right)^{2}=\delta_{0}^{2}, \\
\frac{1}{2} \operatorname{ad}_{x}^{2}\left(\delta_{0} \delta_{2}\right)=\delta_{0} \frac{1}{2} \operatorname{ad}_{x}^{2}\left(\delta_{2}\right)=\delta_{0}^{2} .
\end{gathered}
$$

Subtracting these equations we get

$$
\frac{1}{2} \operatorname{ad}_{x}^{2}\left(\delta_{1}^{2}-\delta_{0} \delta_{2}\right)=0
$$

which implies that in $\mathcal{P}\left(\mathcal{O}_{n}\right)$,

$$
y_{1}^{2}-y_{0} y_{2}=0 .
$$

This relation is also an immediate consequence of the definition of the distinguished homomorphism.

Now using Lemma 4 we can write down a basis of $\mathcal{P}^{p}\left(\mathcal{O}_{n}\right)$ for every $p=0, \ldots, 2 n$ :

$$
\begin{aligned}
y_{2}^{l}, y_{0} y_{2}^{l}, \ldots, y_{0}^{n-l} y_{2}^{l} & \text { for } p=2 l \\
y_{1} y_{2}^{l}, y_{0} y_{1} y_{2}^{l}, \ldots, y_{0}^{n-l-1} y_{1} y_{2}^{l} & \text { for } p=2 l+1 .
\end{aligned}
$$

Since $\delta_{0}^{n+1}=0$ we have

$$
\frac{1}{p !} \operatorname{ad}_{x}^{p}\left(\delta_{0}^{n+1-v_{p}} \delta_{p}\right)=\delta_{0}^{n+1-v_{p}} \delta_{0}^{v_{p}}=\delta_{0}^{n+1}=0,
$$

which implies that

$$
y_{0}^{n+1-v_{p}} y_{p}=0 .
$$

By Lemma 7 this is equivalent to

$$
y_{0}^{n+1-l} y_{2 l-1}=0, \quad y_{0}^{n+1-l} y_{2 l}=0,
$$

and the latter by (11) is equivalent to

$$
y_{0}^{n+1-l} y_{1} y_{2}^{l-1}=0, \quad y_{0}^{n+1-l} y_{2}^{l}=0 .
$$

Again by (11) this implies that for all possible $p_{0}, p_{1}, p_{2}$ with $p_{0}+p_{1}+p_{2}=$ $n+1$,

$$
y_{0}^{p_{0}} y_{1}^{p_{1}} y_{2}^{p_{2}}=0 .
$$

Looking at the basis (12)-(13) we see that (11) and (14) form together a complete system of relations on the generators $y_{0}, y_{1}, y_{2}$, which proves that the map of graded algebras $\mathcal{P}_{n} \rightarrow \mathcal{P}\left(\mathcal{O}_{n}\right), z_{p} \mapsto y_{p}$ for $p=0,1,2$, is well defined and is an isomorphism. The equality $\operatorname{ad}_{x}\left(\delta_{1}\right)=\delta_{0}$ implies $\left\{y_{0}, y_{1}\right\}=y_{0}$ 
and by the construction of $\delta_{1}:=\frac{1}{2} \operatorname{ad}_{x}\left(\delta_{2}\right)$ we have $\left\{y_{0}, y_{2}\right\}=2 y_{1}$. Since

$$
\begin{aligned}
\operatorname{ad}_{x}\left(\left[\delta_{1}, \delta_{2}\right]-\delta_{2}\right) & =\left[\operatorname{ad}_{x}\left(\delta_{1}\right), \delta_{2}\right]+\left[\delta_{1}, \operatorname{ad}_{x}\left(\delta_{2}\right)\right]-\operatorname{ad}_{x}\left(\delta_{2}\right) \\
& =\left[\delta_{0}, \delta_{2}\right]+\left[\delta_{1}, 2 \delta_{1}\right]-\left[\delta_{0}, \delta_{2}\right]=0,
\end{aligned}
$$

we have $\left\{y_{1}, y_{2}\right\}=y_{2}$. This is also an immediate consequence of the distinguished choice of $\delta_{0}, \delta_{1}, \delta_{2}$. Therefore the above isomorphism of graded algebras preserves the Poisson structure.

Corollary 5. $\operatorname{Spec}\left(\mathcal{P}\left(\mathcal{O}_{n}\right)\right)$ admits a structure of a conical Poisson scheme with a hamiltonian infinitesimal algebraic action of $\mathrm{SL}_{2}$, whose moment map is a closed embedding onto the nth infinitesimal neighborhood $Y_{n}$ of the vertex in the nilpotent cone $Y$ in $\mathrm{sl}_{2}^{*}$.

THEOREM 2. The distinguished homomorphism of filtered algebras

$$
\mathcal{U}\left(\mathrm{sl}_{2}\right) /(C-n(n+2)) \rightarrow \mathcal{D}\left(\mathcal{O}_{n}\right)
$$

is surjective with kernel $\left(e^{n+1}\right)$.

Proof. Since the principal symbols $y_{0}, y_{1}, y_{2}$ of the distinguished operators $\delta_{0}, \delta_{1}, \delta_{2}$ generate the associated graded algebra $\mathcal{P}\left(\mathcal{O}_{n}\right)$ of the filtered algebra $\mathcal{D}\left(\mathcal{O}_{n}\right)$, the distinguished operators generate $\mathcal{D}\left(\mathcal{O}_{n}\right)$ as well, which proves the surjectivity of the distinguished homomorphism. Since all maximal ideals in $\mathcal{U}\left(\mathrm{sl}_{2}\right)$ of height two are of the form $\left(e^{n+1}, C-n(n+2)\right), n \geq 0$ ([9, Theorem $4.5(i i)])$, this proves our theorem.

4. Principal symbols and inverse limits. Note that in general the graded Poisson algebra spanned by principal symbols is not functorial with respect to a given commutative algebra. However, graded Poisson algebras spanned by principal symbols of the surjective inverse system $\mathcal{O}_{n+1} \rightarrow \mathcal{O}_{n}$ (when applied object-wise) form a surjective inverse system $\mathcal{P}\left(\mathcal{O}_{n+1}\right) \rightarrow$ $\mathcal{P}\left(\mathcal{O}_{n}\right)$ as well, and we have

$$
\lim _{n} \mathcal{P}\left(\mathcal{O}_{n}\right)=k\left[\left[y_{0}, y_{1}, y_{2}\right]\right] /\left(y_{1}^{2}-y_{0} y_{2}\right) \text {. }
$$

On the other hand, for $\mathcal{O}=k[x]$ we have $\mathcal{P}(\mathcal{O})=k\left[x_{0}, x_{1}\right]$, where $x_{0}$ and $x_{1}$ are principal symbols of the operators $x$ and $d / d x$, respectively. Fix a maximal ideal $\mathfrak{m}=\left(x_{0}, x_{1}\right) \subset \mathcal{P}(\mathcal{O})$ and write $\mathcal{P}(\mathcal{O})_{n}:=\mathcal{P}(\mathcal{O}) / \mathfrak{m}^{n+1}$. Since

$$
\left\{x_{0}, x_{1}\right\}=-1
$$

this ideal and all its powers are not Poisson, so the $\mathcal{P}(\mathcal{O})_{n}$ are not Poisson algebras. However, for $m, n \geq 0$,

$$
\left\{\mathfrak{m}^{m+1}, \mathfrak{m}^{n+1}\right\} \subset \mathfrak{m}^{m+n}
$$

hence the inverse limit

$$
\lim _{n} \mathcal{P}(\mathcal{O})_{n}=k\left[\left[x_{0}, x_{1}\right]\right]
$$

inherits a Poisson structure from $\mathcal{P}(\mathcal{O})$. 
Note that the inverse limits (15), (18) are not graded algebras. This is so because the algebraic action of the multiplicative group scheme $\mathbb{G}_{m}$, equivalent to the grading, in the limit induces only a pro-algebraic action of a formal group, the completion of $\mathbb{G}_{m}$ at the neutral element. This proalgebraic action is equivalent to the action of the Lie algebra of $\mathbb{G}_{m}$ by derivations. Therefore we will call an algebra with a distinguished derivation formally graded. We will call a Poisson algebra which is formally graded as an algebra formally graded Poisson if the distinguished derivation $\varepsilon$ and the Poisson bracket satisfy

$$
\varepsilon(\{f, g\})-\{\varepsilon(f), g\}-\{f, \varepsilon(g)\}=-\{f, g\} .
$$

The algebras (15), (18) are formally graded with distinguished derivations $x_{1} \partial / \partial x_{1}$ and $y_{1} \partial / \partial y_{1}+2 y_{2} \partial / \partial y_{2}$, respectively. It is an interesting fact that these two complete formally graded Poisson $\lim _{n} \mathcal{O}_{n}$-algebras can be related in a canonical way. More precisely, we have the following theorem.

Theorem 3. There exists a unique formal grading preserving Poisson homomorphism of formally graded Poisson algebras making the following diagram commutative:

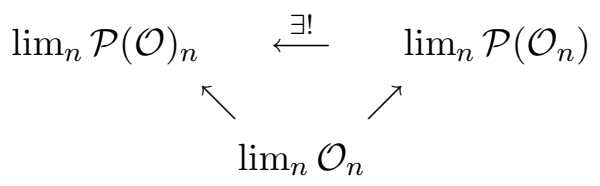

Proof. We will look for a solution of the above problem using the explicit presentation

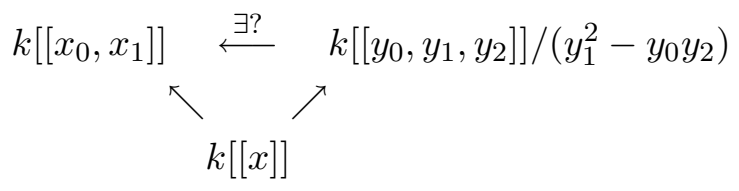

where the structure of $k[[x]]$-algebra is given by $x \mapsto y_{0}$ and $x \mapsto x_{0}$, respectively.

As a homomorphism of $k[[x]]$-algebras the map $k\left[\left[y_{0}, y_{1}, y_{2}\right]\right] /\left(y_{1}^{2}-y_{0} y_{2}\right)$ $\rightarrow k\left[\left[x_{0}, x_{1}\right]\right]$ should be of the form

$$
y_{0} \mapsto x_{0}, \quad y_{1} \mapsto f_{1}, \quad y_{2} \mapsto f_{2},
$$

for some $f_{1}, f_{2} \in k\left[\left[x_{0}, x_{1}\right]\right]$ such that

$$
f_{1}^{2}=x_{0} f_{2},
$$

which implies that

$$
f_{1}=x_{0} g, \quad f_{2}=x_{0} g^{2}
$$

for some $g \in k\left[\left[x_{0}, x_{1}\right]\right]$. 
This is formal grading preserving if and only if $g$ is of degree one, hence of the form

$$
g=h x_{1}
$$

for some $h$ of degree zero, which means that $h \in k\left[\left[x_{0}\right]\right] \subset k\left[\left[x_{0}, x_{1}\right]\right]$. Consequently,

$$
f_{1}\left(x_{0}, x_{1}\right)=x_{0} h\left(x_{0}\right) x_{1}, \quad f_{2}\left(x_{0}, x_{1}\right)=x_{0} h\left(x_{0}\right)^{2} x_{1}^{2} .
$$

This is a Poisson homomorphism if and only if

$$
\left\{x_{0}, f_{1}\right\}=x_{0}, \quad\left\{x_{0}, f_{2}\right\}=2 f_{1}, \quad\left\{f_{1}, f_{2}\right\}=f_{2},
$$

which by (16) and (23) is equivalent to

$$
h=-1 \text {. }
$$

All this means that there exists a unique solution to our problem and it is of the form

$$
y_{0} \mapsto x_{0}, \quad y_{1} \mapsto-x_{0} x_{1}, \quad y_{2} \mapsto x_{0} x_{1}^{2} .
$$

This is a very interesting homomorphism. First of all, it is the completion of a (birational) resolution of singularity in the category of graded Poisson algebras. Geometrically, this resolution is described by the following diagram in the category of conical Poisson varieties:

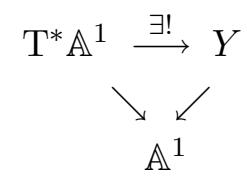

where $Y=\operatorname{Spec}\left(k\left[y_{0}, y_{1}, y_{2}\right] /\left(y_{1}^{2}-y_{0} y_{2}\right)\right)$ is a quadratic cone. The left hand side morphism is a fibration by lines which is mapped into a family of conics degenerating to a double line $\left(y_{0}=0, y_{1}^{2}=0\right)$ on the right hand side. This resolution contracts the line $\left(x_{0}=0\right)$ to the vertex of the cone $Y$ and the scheme-theoretical pre-image of the double line is the line $\left(x_{0}=0\right)$ again.

The following simple proposition means that the above two systems of infinitesimal neighborhoods $\left(\mathbb{A}_{n}^{1}\right.$ of a point in $\mathbb{A}^{1}$ and $Y_{n}$ of the vertex of the nilpotent cone $Y$ ) define the same completion of the cotangent bundle $\mathrm{T}^{*} \mathbb{A}^{1}$.

Proposition 1. There is an isomorphism of conical Poisson formal schemes

$$
\operatorname{colim}_{n}\left(\mathbb{A}_{n}^{1} \times_{\mathbb{A}^{1}} \mathrm{~T}^{*} \mathbb{A}^{1}\right) \cong \operatorname{colim}_{n}\left(Y_{n} \times_{Y} \mathrm{~T}^{*} \mathbb{A}^{1}\right) .
$$

Proof. We have

$$
\begin{aligned}
\mathbb{A}_{n}^{1} \times_{\mathbb{A}^{1}} \mathrm{~T}^{*} \mathbb{A}^{1} & =\operatorname{Spec}\left(k\left[x_{0}, x_{1}\right] /\left(x_{0}^{n+1}\right)\right), \\
Y_{n} \times_{Y} \mathrm{~T}^{*} \mathbb{A}^{1} & =\operatorname{Spec}\left(\mathcal{P}_{n} \otimes_{\mathcal{P}} k\left[x_{0}, x_{1}\right]\right),
\end{aligned}
$$


where

$\mathcal{P}=k\left[y_{0}, y_{1}, y_{2}\right] /\left(y_{1}^{2}-y_{0} y_{2}\right), \quad \mathcal{P}_{n}=k\left[y_{0}, y_{1}, y_{2}\right] /\left(y_{1}^{2}-y_{0} y_{2},\left(y_{0}, y_{1}, y_{2}\right)^{n+1}\right)$, and the grading preserving homomorphism of graded Poisson algebras $\mathcal{P} \rightarrow$ $k\left[x_{0}, x_{1}\right]$ is defined as in (26), i.e.

$$
y_{0} \mapsto x_{0}, \quad y_{1} \mapsto-x_{0} x_{1}, \quad y_{2} \mapsto x_{0} x_{1}^{2} .
$$

Then for non-negative integers $p_{0}, p_{1}, p_{2}$ such that $p_{0}+p_{1}+p_{2}=n+1$,

$$
\left(y_{0}, y_{1}, y_{2}\right)^{n+1} \ni y_{0}^{p_{0}} y_{1}^{p_{1}} y_{2}^{p_{2}} \mapsto(-1)^{p_{1}} x_{0}^{n+1} x_{1}^{p_{1}+2 p_{2}} \in\left(x_{0}^{n+1}\right) .
$$

Therefore $\mathcal{P}_{n} \otimes_{\mathcal{P}} k\left[x_{0}, x_{1}\right] \cong k\left[x_{0}, x_{1}\right] /\left(x_{0}^{n+1}\right)$, which gives the isomorphism of conical Poisson inductive systems

$$
\left(\mathbb{A}_{n}^{1} \times_{\mathbb{A}^{1}} \mathrm{~T}^{*} \mathbb{A}^{1}\right)_{n} \cong\left(Y_{n} \times_{Y} \mathrm{~T}^{*} \mathbb{A}^{1}\right)_{n} .
$$

Since for $m, n \geq 0$,

$$
\left\{\left(x_{0}^{m+1}\right),\left(x_{0}^{n+1}\right)\right\} \subset\left(x_{0}^{m+n+1}\right)
$$

the inverse limit

$$
\lim _{n} k\left[x_{0}, x_{1}\right] /\left(x_{0}^{n+1}\right)=k\left[\left[x_{0}\right]\right]\left[x_{1}\right]
$$

inherits a graded Poisson structure from $k\left[x_{0}, x_{1}\right]$.

5. Infinitesimal-birational duality. Another remarkable property of the above morphism deserves a separate theorem. The following theorem means that the above canonical resolution of singularity together with the Fourier transformation establishes a duality between the above $\mathrm{SL}_{2}$-symmetries of principal symbols of the system of infinitesimal neighborhoods of finite order of a point in the line $\mathbb{A}^{1}$ (infinitesimal picture) and automorphisms of dual principal symbols induced by birational automorphisms of the dual line $\widehat{\mathbb{A}}^{1}$ (birational picture).

Theorem 4 (Magic Diamond Theorem). The following diagram of canonical morphisms in the category of conical Poisson varieties is commutative:

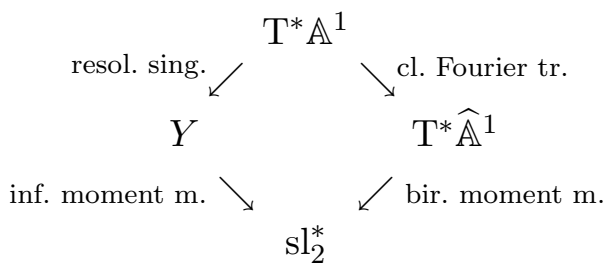

Proof. The left hand side of this diagram is already described in Corollary 5 and Theorem 3 . The composition on the left hand side is

$$
\begin{aligned}
& k\left[z_{0}, z_{1}, z_{2}\right] \rightarrow k\left[x_{0}, x_{1}\right], \\
& z_{0} \mapsto x_{0}, \quad z_{1} \mapsto-x_{0} x_{1}, \quad z_{2} \mapsto x_{0} x_{1}^{2} .
\end{aligned}
$$


The classical Fourier transformation on the right hand side is the following morphism:

$$
\mathrm{T}^{*} \widehat{\mathbb{A}}^{1} \leftarrow \mathrm{T}^{*} \mathbb{A}^{1}, \quad k\left[\widehat{x}_{0}, \widehat{x}_{1}\right] \rightarrow k\left[x_{0}, x_{1}\right], \quad \widehat{x}_{0} \mapsto x_{1}, \quad \widehat{x}_{1} \mapsto-x_{0} .
$$

The infinitesimal form of the rational action of the algebraic group $\mathrm{SL}_{2}$ on $\mathrm{T}^{*} \widehat{\mathbb{A}}^{1}$ canonically induced from the canonical action of $\mathrm{SL}_{2}$ on $\widehat{\mathbb{A}}^{1}$ by birational automorphisms

$$
\left(\begin{array}{ll}
a & b \\
c & d
\end{array}\right) \cdot\left(\widehat{x}_{0}, \widehat{x}_{1}\right)=\left(\frac{a \widehat{x}_{0}+b}{c \widehat{x}_{0}+d},\left(c \widehat{x}_{0}+d\right)^{2} \widehat{x}_{1}\right)
$$

is defined as follows:

$$
\begin{aligned}
& e \mapsto-\frac{\partial}{\partial \widehat{x}_{0}}=\left\{-\widehat{x}_{1},-\right\} \\
& h \mapsto-2 \widehat{x}_{0} \frac{\partial}{\partial \widehat{x}_{0}}+2 \widehat{x}_{1} \frac{\partial}{\partial \widehat{x}_{1}}=\left\{-2 \widehat{x}_{0} \widehat{x}_{1},-\right\}, \\
& f \mapsto \widehat{x}_{0}^{2} \frac{\partial}{\partial \widehat{x}_{0}}-2 \widehat{x}_{0} \widehat{x}_{1} \frac{\partial}{\partial \widehat{x}_{1}}=\left\{\widehat{x}_{0}^{2} \widehat{x}_{1},-\right\}
\end{aligned}
$$

Therefore the moment map associated with this action on $\mathrm{T}^{*} \widehat{\mathbb{A}}^{1}$ is of the form

$$
k\left[z_{0}, z_{1}, z_{2}\right] \rightarrow k\left[\widehat{x}_{0}, \widehat{x}_{1}\right], \quad z_{0} \mapsto-\widehat{x}_{1}, \quad z_{1} \mapsto-\widehat{x}_{0} \widehat{x}_{1}, \quad z_{2} \mapsto-\widehat{x}_{0}^{2} \widehat{x}_{1} .
$$

It is easy to see that its composition with the classical Fourier transformation (30) gives the same as (29), which proves the commutativity of the above square.

REMARK. The moment map on $\mathrm{T}^{*} \widehat{\mathbb{A}}^{1}$ is the restriction of the canonical moment map on $\mathrm{T}^{*} \widehat{\mathbb{P}}^{1}$ associated with the action of the automorphism group of $\widehat{\mathbb{P}}^{1}$. One has the following commutative diagram

$$
\begin{array}{ccc}
\mathrm{T}^{*} \widehat{\mathbb{P}}^{1} \cong & \cong & \widetilde{\mathcal{N}} \\
\text { moment m. } . & \downarrow & \downarrow \text { Springer res. } \\
& \mathrm{sl}_{2}^{*} \stackrel{\text { Killing f. }}{\longleftarrow} \mathcal{N}
\end{array}
$$

where the right hand vertical morphism is the Springer resolution of the nilpotent cone $\mathcal{N} \subset \operatorname{sl}_{2}$ (see [15]). Therefore, by Theorem 4, our canonical resolution as in Theorem 3 is, up to the classical Fourier transformation and the isomorphism provided by the Killing form, essentially nothing but the Springer resolution.

6. Quantization of the infinitesimal-birational duality. The following theorem shows and explains the quantum nature of the above duality. 
Theorem 5 (Quantized Magic Diamond Theorem). There exists the following commutative diagram in the category of almost commutative algebras:

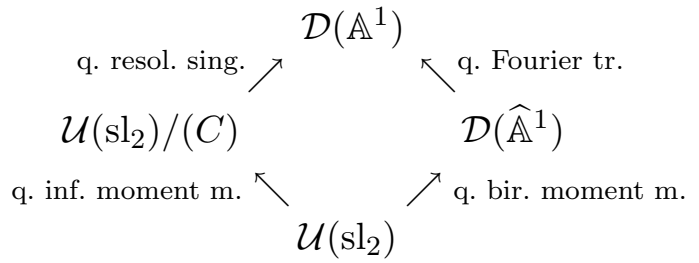

It is a quantization of the Magic Diamond.

Proof. The only nonobvious arrows in this diagram are: the quantized birational moment map on the right hand side and the quantized resolution of singularities on the left hand side. The first one is canonically obtained from the birational action of $\mathrm{SL}_{2}$ on $\widehat{\mathbb{A}}^{1}$ as above, i.e.

$$
e \mapsto-\frac{d}{d \widehat{x}}, \quad h \mapsto-2 \widehat{x} \frac{d}{d \widehat{x}}, \quad f \mapsto \widehat{x}^{2} \frac{d}{d \widehat{x}} .
$$

Composing with the quantum Fourier transformation we get

$$
e \mapsto x, \quad h \mapsto 2 x \frac{d}{d x}+2, \quad f \mapsto-x \frac{d^{2}}{d x^{2}}-2 \frac{d}{d x} .
$$

Note that these operators look exactly like the operators (8) inducing the distinguished differential operators on the $n$th infinitesimal neighborhood of a point in $\mathbb{A}^{1}$, except the strange value $n=-2$. Nevertheless, they satisfy the relation $C=h^{2}+2(e f+f e)=n(n+2)=0$ for $n=-2$, which means that the composite homomorphism factorizes as in the left hand part of the diagram and defines the quantized resolution of singularities on the left hand part. This proves the commutativity of this diagram. Passing to the diagram of associated graded Poisson algebras is equivalent to replacing all differential operators (35) by their principal symbols and all differential operators (34) by their classes modulo differential operators with polynomial coefficients of lower degree. It is easy to see that we obtain the Magic Diamond.

7. Morita equivalences. The strange value $n=-2$ in the latter proof can be related to the Morita equivalence as follows. We have the system of the $n$th infinitesimal neighborhoods $Y_{n}$ of the vertex of the nilpotent cone $Y$ described by the commutative diagram in the category of conical Poisson schemes

$$
\begin{array}{rl}
Y_{n} & \rightarrow Y_{n+1} \\
\downarrow & \\
Y & \downarrow \\
Y & Y
\end{array}
$$


In the above diagram the vertical arrows can be quantized according to Theorems 1 and 2, while horizontal arrows transform into Morita equivalences denoted by $\rightsquigarrow$ in the diagram

$$
\begin{array}{ccc}
\mathcal{D}\left(\mathcal{O}_{n}\right) & \cdots & \mathcal{D}\left(\mathcal{O}_{n+1}\right) \\
\uparrow & \uparrow \\
\mathcal{U}\left(\mathrm{sl}_{2}\right) /(C-n(n+2)) & \rightsquigarrow \mathcal{U}\left(\mathrm{sl}_{2}\right) /(C-(n+1)(n+3))
\end{array}
$$

The upper Morita equivalence is an easy consequence of Corollary 1 and the lower one follows from the Beilinson-Bernstein Theorem [2].

Since there is no homomorphism of the matrix algebras $\mathcal{M}_{n+1} \rightarrow \mathcal{M}_{n}$, surjective homomorphisms of commutative algebras $\mathcal{O}_{n+1} \rightarrow \mathcal{O}_{n}$ cannot induce homomorphisms $\mathcal{D}\left(\mathcal{O}_{n+1}\right) \rightarrow \mathcal{D}\left(\mathcal{O}_{n}\right)$ of the algebras of differential operators. Therefore it is a remarkable fact that homomorphisms $\mathcal{O}_{n+1} \rightarrow \mathcal{O}_{n}$ give rise to Morita equivalences on the level of the algebras $\mathcal{D}\left(\mathcal{O}_{n}\right)$ and surjective homomorphisms on the level of their graded Poisson algebras $\mathcal{P}\left(\mathcal{O}_{n}\right)$.

Since after quantization of Poisson morphisms a twist by Morita equivalences appears, one can expect that in the limit a quantization can be defined only up to Morita equivalence. Indeed, although for any $n \in \mathbb{Z}$ the homomorphism of algebras

$$
\begin{gathered}
\mathcal{U}\left(\mathrm{sl}_{2}\right) /(C-n(n+2)) \rightarrow \mathcal{D}\left(\mathbb{A}^{1}\right), \\
e \mapsto x, \quad h \mapsto 2 x \frac{d}{d x}-n, \quad f \mapsto-x \frac{d^{2}}{d x^{2}}+n \frac{d}{d x},
\end{gathered}
$$

is a quantization of the above resolution of singularity of the nilpotent cone

$$
Y \leftarrow \mathrm{T}^{*} \mathbb{A}^{1}
$$

it has a geometric meaning, related to differential operators on $\mathbb{A}_{n}^{1}$, only for $n \geq 0$. On the other hand, all maximal primitive quotients $\mathcal{U}\left(\mathrm{sl}_{2}\right) /$ $(C-n(n+2))$ for $n \in \mathbb{Z}$ are Morita equivalent to one another (by the Beilinson-Bernstein Theorem) but such a quantization fits into the commutative diagram as in Theorem 5 only for $n=-2$. But then, since $n$ is negative, such a quantization itself has no immediate geometric meaning, although it is Morita equivalent to those quantizations which are related to $\mathcal{D}\left(\mathbb{A}_{n}^{1}\right)$.

\section{References}

[1] J. Becker, Higher derivations and the Zariski-Lipman conjecture, in: Several Complex Variables (Williamstown, MA, 1975), Proc. Sympos. Pure Math. 30, Part 1, Amer. Math. Soc., Providence, RI, 1977, 3-10.

[2] A. Beilinson et J. Bernstein, Localisation de $\mathfrak{g}$-modules, C. R. Acad. Sci. Paris Sér. I Math. 292 (1981), 15-18. 
[3] I. N. Bernstein, I. M. Gelfand and S. I. Gelfand, Differential operators on the cubic cone, Russian Math. Surveys 27 (1972), 169-174.

[4] W. C. Brown, The algebra of differentials of infinite rank, Canad. J. Math. 25 (1973), 141-155.

[5] - Higher derivations on finitely generated integral domains, Proc. Amer. Math. Soc. 42 (1974), 23-27.

[6] W. C. Brown and W.-E. Kuan, Ideals and higher derivations in commutative rings, Canad. J. Math. 24 (1972), 400-415; Addendum, ibid. 29 (1977), 65-70.

[7] P. Brumatti and A. Simis, The module of derivations on a Stanley-Reisner ring, Proc. Amer. Math. Soc. 123 (1995), 1309-1318.

[8] R. Brylinski and B. Kostant, Nilpotent orbits, normality and Hamiltonian group actions, J. Amer. Math. Soc. 7 (1994), 269-298.

[9] S. Catoiu, Ideals of the enveloping algebra U( $\left.\mathrm{sl}_{2}\right)$, J. Algebra 202 (1998), 142-177.

[10] J. Dixmier, Enveloping Algebras, North-Holland Math. Library 14, North-Holland, Amsterdam, 1977.

[11] A. Grothendieck et J. Dieudonné, Éléments de géométrie algébrique IV, Publ. Math. I.H.E.S. 32 (1967).

[12] M. Hochster, The Zariski-Lipman conjecture in the graded case, J. Algebra 47 (1977), 411-424.

[13] Y. Ishibashi, Remarks on a conjecture of Nakai, J. Algebra 95 (1985), 31-45.

[14] —, Nakai's conjecture for invariant subrings, Hiroshima Math. J. 15 (1985), 429436.

[15] J. C. Jantzen, Nilpotent orbits in representation theory, in: Lie Theory, Progr. Math. 228, Birkhäuser Boston, Boston, MA, 2004, 1-211.

[16] A. G. Jones, Rings of differential operators on toric varieties, Proc. Edinburgh Math. Soc. 37 (1994), 143-160.

[17] T. Levasseur, Anneaux d'opérateurs différentiels, in: Lecture Notes in Math. 867, Springer, Berlin, 1981, 157-173.

[18] T. Levasseur and J. T. Stafford, Rings of differential operators on classical rings of invariants, Mem. Amer. Math. Soc. 412 (1991).

[19] J. Lipman, Free derivation modules on algebraic varieties, Amer. J. Math. 87 (1965), $874-898$.

[20] K. R. Mount and O. E. Villamayor, On a conjecture of Y. Nakai, Osaka J. Math. 10 (1973), 325-327.

[21] J. L. Muhasky, The differential operator ring of an affine curve, Trans. Amer. Math. Soc. 307 (1988), 705-723.

[22] I. M. Musson, Differential operators on toric varieties, J. Pure Appl. Algebra 95 (1994), 303-315.

[23] I. M. Musson and M. van den Bergh, Invariants under tori of rings of differential operators and related topics, Mem. Amer. Math. Soc. 650 (1998).

[24] M. Saito and W. N. Traves, Differential algebras on semigroup algebras, in: Contemp. Math. 286, Amer. Math. Soc., 2001, 207-226.

[25] —, - Finite generation of rings of differential operators of semigroup algebras, J. Algebra 278 (2004), 76-103.

[26] A. Schreiner, On a conjecture of Nakai, Arch. Math. (Basel) 62 (1994), 506-512.

[27] B. Singh, Differential operators on a hypersurface, Nagoya Math. J. 103 (1986), 67-84.

[28] K. E. Smith and M. van der Bergh, Simplicity of rings of differential operators in prime characteristic, Proc. London Math. Soc. 75 (1997), 32-62.

[29] S. P. Smith and J. T. Stafford, Differential operators on an affine curve, Proc. London Math. Soc. 56 (1988), 229-259. 
[30] W. N. Traves, Differential operators on monomial rings, J. Pure Appl. Algebra 136 (1999), 183-187.

[31] —, Nakai's conjecture for varieties smoothed by normalization, Proc. Amer. Math. Soc. 127 (1999), 2245-2248.

[32] -, Differential operators and Nakai's conjecture, Ph.D. thesis, Univ. Toronto, 1998.

[33] J. R. Tripp, Differential operators on Stanley-Reisner rings, Trans. Amer. Math. Soc. 349 (1997), 2507-2523.

[34] J.-P. Vigué, Opérateurs différentiels sur les cônes normaux de dimension 2, C. R. Acad. Sci. Paris Sér. A 278 (1974), 1047-1050.

Institute of Mathematics

Polish Academy of Sciences

Śniadeckich 8

00-956 Warszawa, Poland
Institute of Mathematics

University of Warsaw

Banacha 2

02-097 Warszawa, Poland

E-mail: maszczyk@mimuw.edu.pl

Received 25 January 2005;

in revised form 17 February 2006 